\title{
ORIGINAL ARTICLE HIGH SALT INTAKE IMPLICATIONS AS RISK FACTOR FOR HYPERTENSION AND COMPARISON OF THREE SALT ESTIMATION METHODS- FINDINGS FROM ISLAMABAD, PAKISTAN
}

\author{
Muhammad Arif Nadeem Saqib ${ }^{1}$, Ibrar Rafique ${ }^{2}$, Muhammad Ansar $^{3}$, Tayyaba Rahat ${ }^{2}$ \\ ${ }^{1}$ National Skills University, Islamabad, Pakistan, ${ }^{2}$ Health Research Institute, National Institutes of Health (Ex-PHRC), Islamabad, \\ Pakistan, ${ }^{3}$ Quaid-e-Azam University, Islamabad, Pakistan
}

\begin{abstract}
Objectives: The study was designed to estimate daily salt intake, its discretionary use in healthy individuals and to validate three common methods for salt estimation in Pakistani population. Methodology: Information on demography and discretionary salt use was collected from healthy adults ( $>18$ years) along with a blood sample, spot and 24 hour urine samples. Sodium, chloride, potassium levels and serum creatinine were measured using standard methods. For daily salt estimation, three common methods i.e. INTERSALT, Tanaka and Kawasaki were validated for their applicability in local settings.

Results: Overall $24 \mathrm{~h}$ sodium excretion was $158 \mathrm{mmol} / \mathrm{l}$ indicating intake of $8.64( \pm 4.43)$ grams salt per day which was significantly associated with male gender $(\mathrm{p} .<0.004)$ and adding salt during cooking (p. $<0.0001)$. Most $(73 \%)$ of the participants know about hazardous effects of high salt intake, however, only $25 \%$ consider important to lower salt intake. None of three methods i.e. INTERSALT (bias: -19.64; CCC -0.79), Tanaka (bias: 167.35; CCC -0.37) and Kawasaki (bias: -42.49, CCC -0.79) showed any agreement between measured and estimated 24 hour sodium.

Conclusion: Daily intake of salt was high which increases the risk for hypertension. Comparison of methods for estimation revealed that none of the three methods could be used for estimating daily intake of salt in local settings of Pakistan.
\end{abstract}

Keywords: Salt intake, NCDs, salt estimation, 24 hours urine

Citation: Saqib MAN, Rafique I, Ansar M, Rahat T. High Salt Intake Implications as Risk Factor for Hypertension and Comparison of Three Salt Estimation Methods- Findings from Islamabad, Pakistan. Pak Heart J. 2021;54(04):309-

314. DOI: https://doi.org/10.47144/phj.v54i4.2156

\section{INTRODUCTION}

High intake of salt is associated with increased risk of hypertension, heart diseases and gastric cancer. ${ }^{1}$ In most of the countries, average daily salt intake ranges 9-12 g/day that is much higher than recommended i.e. $<5 \mathrm{~g} /$ day. $^{2}$ It has been reported that noncommunicable diseases (NCDs) burden could be decreased in the population by lowering salt intake. This will help in reducing the burden of NCDs globally with low cost. ${ }^{3}$ Keeping in view the importance, the member states of WHO agreed to usefulness of reducing salt intake, the WHO member states have agreed for $30 \%$ decrease in sodium intake till 2025. ${ }^{4}$

Estimation of daily salt is an important step to observe the level of sodium intake among general population which can provide the way forward to public health initiatives. For daily salt estimation, the 24 hour urine collection is the standard method for salt estimation but is laborious and troublesome especially for healthy individuals. In order to overcome hurdles associated with 24 hour urine collection, different mathematical equations have been formulated using spot urine. ${ }^{5,6}$ Although spot test does not provide accurate prediction for 24 hour sodium, however, this test can be used for population estimates and can provide adequate information for monitoring. ${ }^{7}$

Despite the rising burden of NCDs, especially hypertension and cardiovascular diseases (CVDs) in Pakistan, ${ }^{8}$ information on the daily salt intake and its discretionary use was not available. Similarly, to our best knowledge, there was no report available addressing the issue of daily salt estimation in the local population. Therefore, this study was planned to estimate daily salt intake as implication for risk factor for hypertension and comparison of methods used for salt estimation using spot urine.

\section{METHODOLOGY}

This was a descriptive cross-sectional study conducted at Islamabad. Total of 120 healthy subjects aged more than 18 years were enrolled using non-probability convenient sampling. Those who had a history of hypertension or currently hypertensive, taking any medications, difficulty in collecting urine and had pregnancy were excluded. It was assured that participants were healthy and were not suffering from 
fever, diarrhea or any other infections at the time of sample collection.

Information about demographics, discretionary salt use, awareness about the hazards of increased salt intake and their attitude for salt control was collected from the residents of Islamabad using a pre-designed questionnaire. Weight and height were measured as per standard protocol. The data was collected by a trained data collector who also briefed the participants about the process of sample collection. All testing was done at Excel Lab (Pvt.) Ltd which is registered in the Programs for Excellence in Laboratory testing of the College of American Pathologists. The Excel Lab (Pvt) Ltd has sample collection points in the entire city of Islamabad.

After enrollment, data of each participant registered in Excel lab (Pvt) Ltd database along with detail of tests to be carried out which automatically generated a unique identification number along with barcodes for each test. These barcodes were placed on spot and 24hour urine collection containers and gel tube for a blood sample.

Two labeled collection containers (each separate for 24 hour urine and spot urine sample) were given to the participants. All participants were briefed about the process of collection of both spot and $24 \mathrm{~h}$ urine collection. They were asked to discard the first urine sample at 8.00 am on starting day while including the first sample on the second day at $8.00 \mathrm{am}$.

The participants collected 24 hour urine samples as per convenience but preferably on the weekend. Majority of samples were collected on the weekend where the participants started their urine collection on Saturday morning till Sunday morning. The blood samples were collected during the urine collection for estimation of serum creatinine and electrolytes. The participants living in close vicinity of the Excel Labs collection points dropped their sample at their nearest point in the morning after completing 24 hour collection of urine. The participants provided second morning urine sample as a spot sample when coming to Laboratory. From these collection points, samples were immediately transported to main laboratory for testing.

Those participants, who lived far from collection points or reluctant to visit collection point, data collectors themselves visited their home in the early morning. They also provided second morning urine sample as a spot sample. This helped us to assure that second-morning sample was taken as spot sample and was not part of 24 hour urine sample. The data collectors picked samples from their home and directly dropped at the main lab for analysis.
Each 24 hour sample was assessed for its completeness. Those samples having volume $<0.5 \mathrm{~mL}$ and reported missed collection were discarded. Similarly samples collected outside the range of $24 \pm 2 \mathrm{~h}$ were also excluded. All samples were analyzed on the day of their collection. The potassium, sodium, and chloride were tested using electrode method while serum creatinine was done using the kinetic Jaffe reaction along with controls.

Ethics Statement: Institutional Bioethics Committee of Pakistan Health Research Council provided ethical clearance. The research work carried according to the Helsinki Declaration and all participants gave written informed consent for their participation.

Statistical analysis: The data was entered in MS Excel and analyzed using SPSS 21 (SPSS \& IBM, Inc, Chicago, Illinois, USA) for analysis. Tukey test ${ }^{9}$ was used to check outliers at either side, categorized as 'outside' or 'far out' values. The outside and far out values were removed from data. Finally data of 106 participants was analyzed.

Gender wise difference between demographic characteristics and various analytes was determined using independent sample t-test while discretionary salt use and its association with risk factors was assessed by multivariate logistic regression.

The intake of salt on daily basis was calculated by the formula described elsewhere ${ }^{10}$ while 24 hour sodium in urine was estimated using Tanaka ${ }^{5}$, Kawasaki ${ }^{6}$, and INTERSALT $^{11}$ methods. Paired t-test was applied to assess the difference between 24 hour measured and estimated salt intake. Correlation methods (concordance correlation co-efficient and intraclass consistency was used for determining the validity of three methods for estimating 24 hour sodium by visualizing on scatter plot. Systematic bias between measured and estimated sodium was assessed by Bland and Altman plot. P-value $(<0.05)$ was considered statistically significant.

\section{RESULTS}

Out of 120 participants, 92 (77\%) were males and 28 $(23 \%)$ were females. The mean BMI (Body mass index) was $39( \pm 11.47)$ and mean age was $26.5( \pm 5.0)$ years. The mean volume of 24 hour urine was 1501 $( \pm 884) \mathrm{ml} /$ day. Similarly, mean serum creatinine level was $0.88( \pm .15) \mathrm{mg} / \mathrm{dl}$ ranged between 0.5 to $1.2 \mathrm{mg} / \mathrm{dl}$ and 24-hour urine creatinine clearance was 107.9 $( \pm 38.8) \mathrm{ml} / \mathrm{min}$ indicating normal kidney function. There was no significant difference between male and female for these analytes except sodium which was higher in females as compared to males $(\mathrm{p}<0.002)$ 
Mean 24 hour sodium concentration was $158 \mathrm{mmol} / \mathrm{l}$, which is almost equal to $8.64 \pm 4.43 \mathrm{~g}$ salt per day. There was a significant association between discretionary salt use with male gender $(p<0.004)$ and adding salt during cooking ( $p<0.0001)$. However, there was no difference for age, BMI, adding salt on dinning, took salty food from restaurant, and knowledge about the impact of high salt on health (Table 1).

Table 1: Association of discretionary salt with different risk factors

\begin{tabular}{|l|c|c|}
\hline Description & P-Value & $\begin{array}{c}\text { Odds Ratio } \\
\text { 95\% Confidence Interval }\end{array}$ \\
\hline Age & 0.374 & $\begin{array}{c}2.04 \\
(0.63-6.65)\end{array}$ \\
\hline Gender (Male) & 0.004 & $\begin{array}{c}5.31 \\
(1.88-15.02)\end{array}$ \\
\hline BMI & 0.752 & $\begin{array}{c}1.66 \\
(0.58-4.74)\end{array}$ \\
\hline $\begin{array}{l}\text { Adding Salt } \\
\text { during Cooking }\end{array}$ & $<0.001$ & $\begin{array}{c}0.97 \\
(0.94-1.00)\end{array}$ \\
\hline $\begin{array}{l}\text { Adding Salt } \\
\text { during Dinning }\end{array}$ & 0.584 & $\begin{array}{c}0.66 \\
(0.25-1.73)\end{array}$ \\
\hline $\begin{array}{l}\text { Eaten salty food } \\
\text { from restaurant, }\end{array}$ & 0.842 & $\begin{array}{c}1.46 \\
(0.48-4.41)\end{array}$ \\
\hline $\begin{array}{l}\text { Knowledge about } \\
\text { the impact of high } \\
\text { salt on health }\end{array}$ & 0.126 & $\begin{array}{c}0.487 \\
(0.150-1.578)\end{array}$ \\
\hline
\end{tabular}

A majority (71\%) of the participants were using salt in their daily food while more than half were also adding extra salt at the dining table. When inquired from participants that how they categorized themselves regarding salt consumption, $91 \%$ believed it as per their need. Most (73\%) of them were aware about the impact of extra use of salt on their health. Among all, $71 \%$ knew that it might cause hypertension and $11 \%$ associate this with bone diseases. Only few participants were aware that high level of salt might also cause gastric cancer and kidney stones. Twentyfive per cent of participants believed that reduction in daily salt intake is important for their health while others considered this either less important $(57 \%)$ or gave no importance $(17 \%)$.

Tanaka and Kawasaki equations showed significant difference between measured and estimated 24 hour sodium. However, the difference between measured and estimated 24 hour sodium using INTERSALT method was insignificant (p. 0.09). Correlation analysis showed that none of three methods i.e. INTERSALT (bias: -19.64; CCC -0.79), Tanaka (bias: 167.35; CCC -0.37) and Kawasaki (bias: -42.49, CCC -0.79) had showed agreement between estimated and measured 24 hour sodium. (Table 2, Figure 1). Similarly, the Bland Altman plot also indicated an inconsistent pattern between estimated and measured 24 hour sodium (Figure 2).
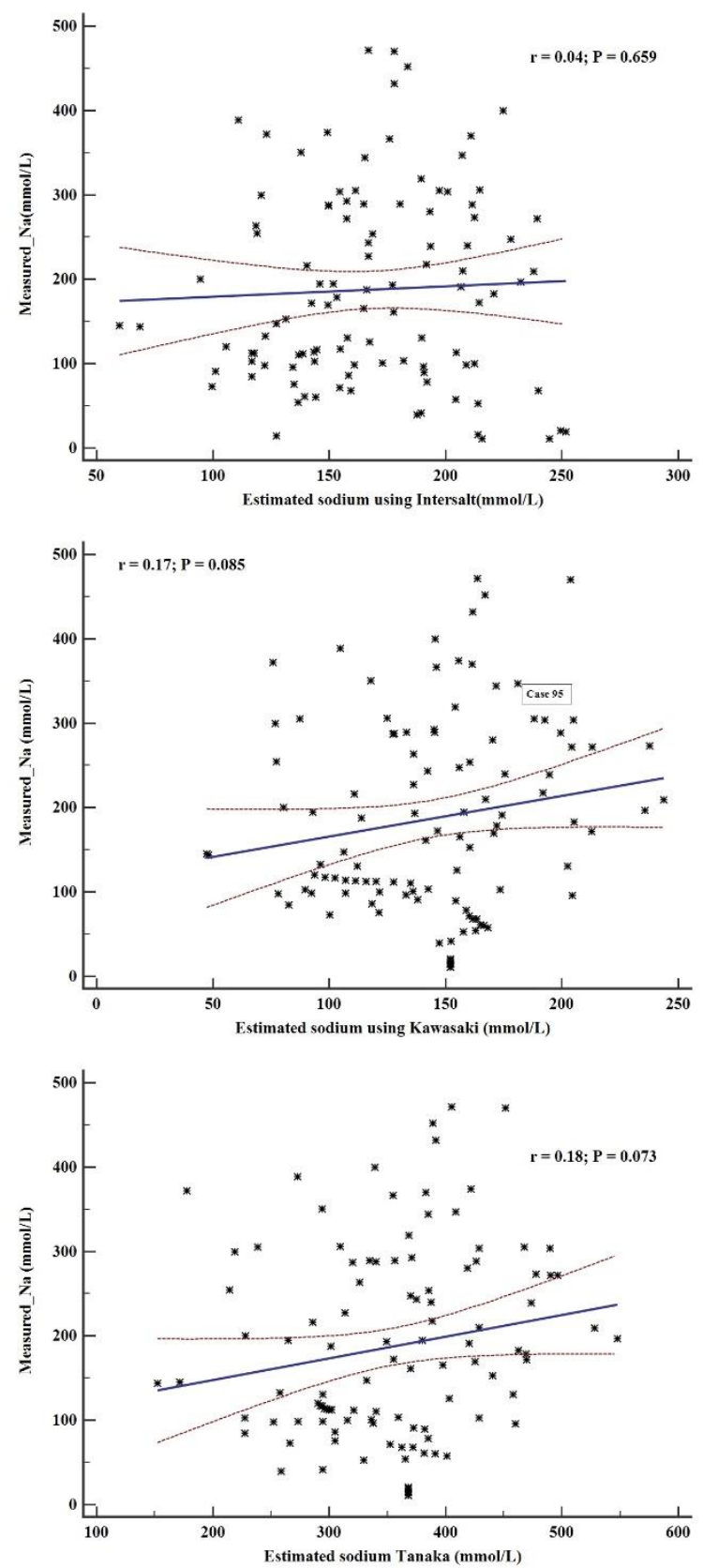

Figure 2: Bland Altman plot of measured 24-h urine sodium excretion versus INTERSALT (A), Kawasaki (B) and Tanaka (C) method estimated 24-h (mmol/L)

The difference between measured and estimated was all estimated values minus the measured values. The mid red line is representing the mean difference or bias between measured and estimated values while the dashed line represents $95 \%$ limits of agreement of the mean difference $( \pm 1.96 S D)$ 
Table 2: Comparison of measured $(24 \mathrm{~h})$ and estimated sodium

\begin{tabular}{|l|l|l|l|l|}
\hline Description & Mean Difference & $\begin{array}{l}\text { Stand } \\
\text { Error }\end{array}$ & $\begin{array}{l}\text { Concordance Correlation } \\
\text { Coefficient }\end{array}$ & $\begin{array}{l}\text { Intraclass Correlation } \\
\text { Coefficient }\end{array}$ \\
\hline Measured Na (mmol/L) & Reference & Reference & Reference & Reference \\
\hline Intersalt $(\mathbf{m m o l} / \mathbf{L})$ & -19.64 & 11.65 & $-0.79(-0.84$ to -0.69$)$ & $-0.63(-0.74$ to -0.50$)$ \\
\hline Tanaka $(\mathbf{m m o l} / \mathbf{L})$ & $167.35^{*}$ & 12.31 & $-0.37(-0.53$ to -0.19$)$ & $-0.33(-0.49$ to -0.15$)$ \\
\hline Kawasaki $(\mathbf{m m o l} / \mathbf{L})$ & $-42.49^{*}$ & 11.16 & $-0.79(-0.85$ to -0.70$)$ & $-0.67(-0.76$ to -0.55$)$ \\
\hline
\end{tabular}

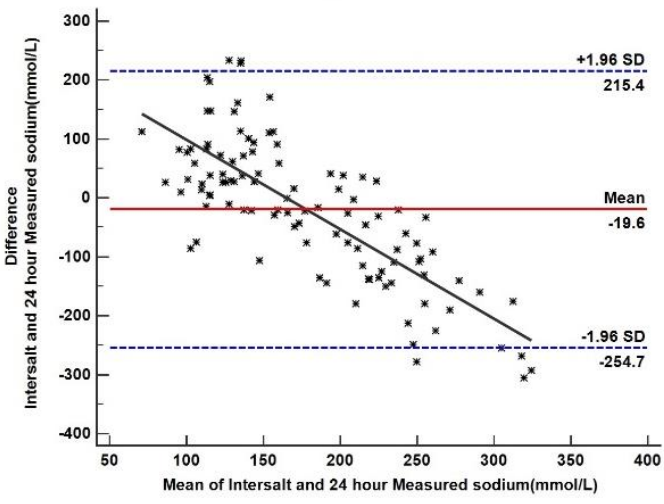

B

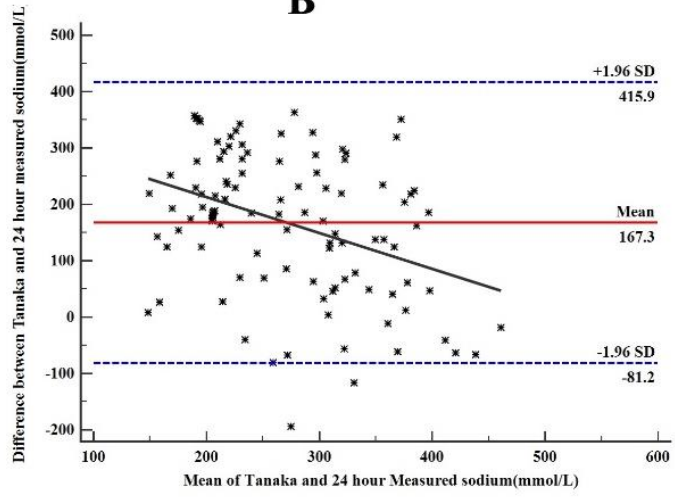

C

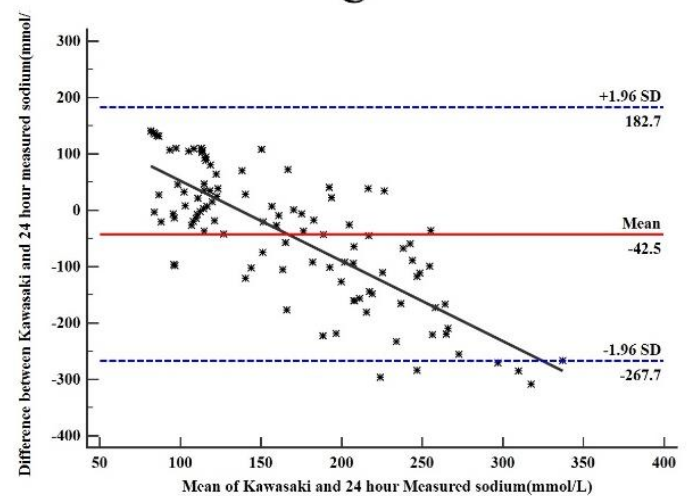

Figure 2: Bland Altman plot of measured 24-hour urine sodium excretion versus INTERSALT (A), Kawasaki (B) and Tanaka (C) method estimated 24-h (mmol/L).

The difference between measured and estimated was all estimated values minus the measured values. The mid red line is representing the mean difference or bias between measured and estimated values while the dashed line represents $95 \%$ limits of agreement of the mean difference $( \pm 1.96 S D)$

\section{DISCUSSION}

In this study, mean salt intake was 8.4 grams per day and was significantly associated with male gender and adding extra salt during dining at the table. The WHO recommends that salt intake should be less 5 grams per day. ${ }^{12}$ High salt intake has been associated with different diseases including hypertension, gastric cancer, kidney stones and bone deformities. ${ }^{13}$ Similar studies from India, Bangladesh and China also reported high salt intake of $10.98,17$ and 11.8 grams per day, respectively. ${ }^{14,15}$ High daily salt intake is also common in developed countries like in Australia where the mean daily intake of salt was 8.8 grams/day. ${ }^{16}$
In Southeast Asia, excess salt intake is part of culinary culture, which results in increased consumption of daily salt in this region. ${ }^{17}$ However, the habit of adding salt during dinning is dangerous. Our study showed an association of discretionary salt use with male gender which is consistent with previous studies. ${ }^{18}$ It was reported that high intake of salt in men might be due to high intake of food as compared to women. ${ }^{19}$ Another reason might be outside eating which was more in men as compared to women in Pakistan.

Keeping in view the impact of high salt intake, the WHO members states agreed to reduce this by a relative of $30 \%$ by $2025 .^{12}$ Many countries especially the developed ones have started different initiatives to reduce the daily salt intake. It has been shown that 
initiatives taken by the governments for the population-level reduction of salt intake were more useful. ${ }^{20}$ Pakistan is facing the dual burden of noncommunicable and communicable diseases. ${ }^{21}$ It was reported that NCDs risk factors like tobacco, physical inactivity and unhealthy diet were common and there was a gradual increase in the burden of different NCDs especially diabetes, hypertension, and cardiovascular diseases. $^{8,22}$ The current finding of high daily salt intake is an addition to existing information on prevalence of different NCDs risk factors. Being the signatory of WHO treaty to lower the intake of salt in population, the Government of Pakistan must take urgent measures at policy level for reducing salt intake in the population. This will eventually help in reducing the burden of NCDs.

Estimating the salt intake (daily) is important to estimate the usage and monitor the impact of different interventions. The 24 hour urine is the standard method for estimating the intake of salt measuring the excretion of sodium in the urine. However, this is long and laborious and is impractical at population level. To overcome this issue, INTERSALT, Tanaka and Kawasaki methods ${ }^{5}, 6,11$ were formulated on concentrations of spot urine potassium, sodium, and creatinine along with gender, height, weight, and age.

The INTERSALT equation was derived using data of spot urine sodium concentration and 24 hour sodium excretion urine which was collected from 52 different populations belonging to 32 countries. ${ }^{11}$ On the other hand, both Kawasaki and Tanaka methods were derived using data of Japanese population. ${ }^{5,6}$ Different studies have validated these methods to estimate daily salt intake using spot urine but their utility varied from population to population. ${ }^{7,} 10$ Additionally, these methods are inadequate to measure the intake of salt (daily) using spot urine sample but still excellent option for estimating at the population level. ${ }^{10}$

The applicability of INTERSALT for estimating intake of salt was reported from different Western and Asian countries. ${ }^{20}$ It was shown that INTERSALT could be useful for estimating intake of salt by spot urine in the Asian population. ${ }^{23}$ In our study, the difference between estimated and measured sodium using INTERSALT ${ }^{11}$ method was insignificant but the correlation analysis did not show any agreement, therefore, we do not support this notion. On the other hand, both Kawasaki and Tanaka methods had a significant difference between the means of estimated and measured 24 hour sodium. The correlation analysis also showed disagreement between estimated and measured 24 hour sodium excretion for both methods. Our study findings are consistent to previous reports indicating that probably these methods could not be used for all populations and there is need to make local equations. ${ }^{10,24}$

The value of creatinine concentration in a spot urine sample is important in predicting daily salt intake. Creatinine is produced during metabolization of creatinine phosphate in muscles. Hence its excretion in urine is dependent on body mass and protein intake. Similarly, creatinine excretion also varies among individuals depending on gender, age groups and other demographic features. The sodium to creatinine ratio might not depend on difference at population level as there is low protein intake in developing countries. ${ }^{25}$ Also, Kawasaki and Tanaka's methods derived using data of the Japanese population might not truly representing our population.

In summary, our study showed that salt intake (daily) is high though the people were aware of its impact on their health. The analysis also showed that the three methods i.e. Tanaka, Kawasaki and INTERSALT for 24 hour estimation are not applicable for Pakistani population. Our study provided a base for conducting large scale studies to formulate accurate equation for estimation of daily salt intake for local population. Besides this, interventional studies are also required for evaluating the possible interventions for behavior change in the population.

Our study has potential limitations like having fewer samples, lack of diversity with respect to ethnicity but still provides the valuable information regarding the validity of three methods for the estimating salt intake.

\section{CONCLUSION}

High daily salt intake was observed which increased the risk for hypertension. The comparison between three methods of estimation revealed that none of the three methods could be used for estimating daily intake of salt using spot urine in local settings of Pakistan.

\section{AUTHORS' CONTRIBUTION:}

The MANS conceptualize and designed the study, secure the funding and revise the manuscript, IR drafted the project and manuscript, MA interpreted the data and revise the article with intellectual content, TR performed the statistical analysis and interpreted results.

Conflict of interest: Authors declared no conflict of interest.

Acknowledgment: We are thankful to the Pakistan Health Research Council (PHRC) for funding, Excel labs staff for their dedication to test the samples timely especially Mr. Abdul Waheed, Manager Operations for his continuous support through the project. We are also obliged to all participants who voluntarily provided samples especially 24 hour urine sample. We are highly 
thankful to Mr. Saeed Ahmed Shahid for coordinating with the participants for collection of samples.

\section{REFERENCES}

1. He FJ, Tan M, Ma Y, MacGregor GA. Salt Reduction to Prevent Hypertension and Cardiovascular Disease: JACC State-of-the-Art Review. J Am Coll Cardiol. 2020;75(6):632-47.

2. Dotsch-Klerk M, Goossens WP, Meijer GW, van het Hof KH Reducing salt in food; setting product-specific criteria aiming at a salt intake of $5 \mathrm{~g}$ per day. Eur. J. Clin. Nutr. 2015;69(7):799-804.

3. Al Jawaldeh A, Rafii B, Nasreddine L. Salt intake reduction strategies in the Eastern Mediterranean Region. East Mediterr Health J. 2019;24(12):1172-80.

4. Santos JA, Tekle D, Rosewarne E, Flexner N, Cobb L, AlJawaldeh A, Kim WJ, et al. A Systematic Review of Salt Reduction Initiatives Around the World: A Midterm Evaluation of Progress Towards the 2025 Global Non-Communicable Diseases Salt Reduction Target. Adv Nutr. 2021;12(5):1768-80.

5. Tanaka T, Okamura T, Miura K, Kadowaki T, Ueshima H, Nakagawa $\mathrm{H}$, et al. A simple method to estimate populational 24$\mathrm{h}$ urinary sodium and potassium excretion using a casual urine specimen. J Hum Hypertens. 2002;16(2):97-103.

6. Kawasaki T, Itoh K, Uezono K, Sasaki H. A simple method for estimating $24 \mathrm{~h}$ urinary sodium and potassium excretion from second morning voiding urine specimen in adults. Clin. Exp. Pharmacol. Physiol. 1993;20(1):7-14.

7. McLean R, Williams S, Mann J. Monitoring population sodium intake using spot urine samples: validation in a New Zealand population. J. Hum. Hypertens. 2014;28(11):657-62.

8. Saqib MAN, Rafique I, Qureshi H, Munir MA, Bashir R, Arif BW, et al. Burden of Tobacco in Pakistan: Findings From Global Adult Tobacco Survey 2014. Nicotine Tob. Res. 2018;20(9):113843.

9. Tukey JW. Exploratory Data Analysis. Reading, Massachusetts: Addison-Wesley; 1977.

10. Peng Y, Li W, Wang Y, Chen H, Bo J, Wang X, et al. Validation and Assessment of Three Methods to Estimate 24-h Urinary Sodium Excretion from Spot Urine Samples in Chinese Adults. PloS One. 2016;11(2):e0149655.

11. Brown IJ, Dyer AR, Chan Q, Cogswell ME, Ueshima H, Stamler $\mathrm{J}$, et al. Estimating 24-hour urinary sodium excretion from casual urinary sodium concentrations in Western populations: the INTERSALT study. Am. J. Epidemiol. 2013;177(11):1180-92.

12. Mills KT, Bundy JD, Kelly TN, Reed JE, Kearney PM, Reynolds $\mathrm{K}$, et al. Global Disparities of Hypertension Prevalence and Control: A Systematic Analysis of Population-Based Studies From 90 Countries. Circulation. 2016;134(6):441-50.

13. Tiyasatkulkovit W, Aksornthong S, Adulyaritthikul P, Upanan P, Wongdee K, Aeimlapa R, et al. Excessive salt consumption causes systemic calcium mishandling and worsens microarchitecture and strength of long bones in rats. Sci Rep. 2021;11(1):1850.

14. Zaman MM, Choudhury SR, Ahmed J, Khandaker RK, Rouf MA, Malik A. Salt Intake in an Adult Population of Bangladesh. Glob Heart. 2017;12(3):265-6.

15. Xu J, Wang M, Chen Y, Zhen B, Li J, Luan W, et al. Estimation of salt intake by 24-hour urinary sodium excretion: a crosssectional study in Yantai, China. BMC Public Health. 2014;14:136

16. Land MA, Webster J, Christoforou A, Praveen D, Jeffery P, Chalmers J, et al. Salt intake assessed by $24 \mathrm{~h}$ urinary sodium excretion in a random and opportunistic sample in Australia. BMJ Open. 2014;4(1):e003720.

17. Vijayaraghavan K, McCullough PA, Singh B, Gupta M, Enas E, Mohan V, et al. Cardiometabolic-Renal Disease in South Asians: Consensus Recommendations from the Cardio Renal Society of America. Cardiorenal Med. 2019;9(4):240-51

18. Brouillard AM, Kraja AT, Rich MW. Trends in Dietary Sodium Intake in the United States and the Impact of USDA Guidelines: NHANES 1999-2016. Am J Med. 2019;132(10):1199-1206.e5.

19. Ahmed $\mathrm{H}$, Thaver IH. Hypertension and obesity in community of Nain-Sukh. J Pak Med Assoc. 2020;70(4):482-7.

20. McLaren L, Sumar N, Barberio AM, Trieu K, Lorenzetti DL, Tarasuk V, et al. Population-level interventions in government jurisdictions for dietary sodium reduction. The Cochrane database of systematic reviews. Int J Epidemiol. 2016;9:CD010166.

21. Country Cooperation Strategy. Health Situation. Pakistan. World Health Organization. 2018; http://apps.who.int/iris/bitstream/handle/10665/136607/ccsbrief pak en.pdf;jsessionid=5D5AC8B53D20CBAE87E22FA486E62 662? sequence $=1$. Accessed 22 ${ }^{\text {nd }}$ October 2018.

22. Rafique I, Saqib MAN, Munir MA, Qureshi H, Rizwanullah, Khan SA, Fouad H. Prevalence of risk factors for noncommunicable diseases in adults: key findings from the Pakistan STEPS survey. East Mediterr Health J. 2018;24(1):3341

23. Whitton C, Gay GM, Lim RB, Tan LW, Lim WY, van Dam RM. Evaluation of Equations for Predicting 24-Hour Urinary Sodium Excretion from Casual Urine Samples in Asian Adults. J Nutr. 2016;146(8):1609-15.

24. Ma W, Yin X, Zhang R, Liu F, Yang D, Fan Y, et al. Validation and Assessment of Three Methods to Estimate 24-h Urinary Sodium Excretion from Spot Urine Samples in High-Risk Elder Patients of Stroke from the Rural Areas of Shaanxi Province. Int J Environ Res Public Health. 2017;14(10):1211

25. Bourdoux P. Evaluation of the iodine intake: problems of the iodine/creatinine ratio--comparison with iodine excretion and daily fluctuations of iodine concentration. Exp Clin Endocrinol Diabetes. 1998;106(Suppl 3):S17-20.

\section{Address for Correspondence:}

Dr. Ibrar Rafique, Research Officer, Pakistan Health Research Council, Shahrah-e-Jamhuriat, Constitution Avenue, G-5/2, Islamabad.

Email: ibrarpmrc@gmail.com 